\title{
ARTICLE
}

\section{STEM CELL BIOLOGY}

\section{The balance between the intronic miR-342 and its host gene Evl determines hematopoietic cell fate decision}

\author{
Friederike Herbst $\mathbb{D}^{1}$ - Tonio J. L. Lang ${ }^{1,2}$ - Elias S. P. Eckert ${ }^{1,3}$ - Peer Wünsche ${ }^{1,3}$ - Alexander A. Wurm ${ }^{4,5,6}$. \\ Tim Kindinger ${ }^{1} \cdot$ Karin Laaber $\mathbb{D}^{1,3} \cdot$ Shayda Hemmati ${ }^{1,3} \cdot$ Agnes Hotz-Wagenblatt $\mathbb{D}^{7} \cdot$ Oksana Zavidij ${ }^{1}$. \\ Anna Paruzynski ${ }^{8} \cdot$ Junyan $\mathrm{Lu}^{9} \cdot$ Christof von Kalle $\mathrm{C}^{10,11,12} \cdot$ Thorsten Zenz $\mathbb{D}^{13} \cdot$ Christoph Klein $\mathbb{( D}^{14}$. \\ Manfred Schmidt ${ }^{10,11} \cdot$ Claudia R. Ball $^{5,6,15} \cdot$ Hanno Glimm ${ }^{1,5,6,15}$
}

Received: 10 September 2018 / Revised: 6 April 2021 / Accepted: 27 April 2021 / Published online: 21 May 2021

(c) The Author(s) 2021. This article is published with open access

\begin{abstract}
Protein-coding and non-coding genes like miRNAs tightly control hematopoietic differentiation programs. Although miRNAs are frequently located within introns of protein-coding genes, the molecular interplay between intronic miRNAs and their host genes is unclear. By genomic integration site mapping of gamma-retroviral vectors in genetically corrected peripheral blood from gene therapy patients, we identified the EVL/MIR342 gene locus as a hotspot for therapeutic vector insertions indicating its accessibility and expression in human hematopoietic stem and progenitor cells. We therefore asked if and how EVL and its intronic miRNA-342 regulate hematopoiesis. Here we demonstrate that overexpression (OE) of Evl in murine primary $\mathrm{Lin}^{-} \mathrm{Scal}^{+} \mathrm{cKit}^{+}$cells drives lymphopoiesis whereas miR-342 $\mathrm{OE}$ increases myeloid colony formation in vitro and in vivo, going along with a profound upregulation of canonical pathways essential for B-cell development or myelopoietic functions upon Evl or miR-342 OE, respectively. Strikingly, miR-342 counteracts its host gene by targeting lymphoid signaling pathways, resulting in reduced pre-B-cell output. Moreover, EVL overexpression is associated with lymphoid leukemia in patients. In summary, our data show that one common gene locus regulates distinct hematopoietic differentiation programs depending on the gene product expressed, and that the balance between both may determine hematopoietic cell fate decision.
\end{abstract}

\section{Introduction}

Hematopoiesis generates differentiated blood cells from stem and progenitor cells tightly controlled and adjusted to the needs of the organism by cell intrinsic and extrinsic factors [1-3]. Robustness of lineage differentiation is supported by microRNAs (miRNA) [4] on a posttranscriptional level. These small non-coding RNAs are either intronic or intergenic and mainly transcribed by Pol II leading to 21-24-bp double-stranded RNAs after several cleavage

These authors contributed equally: Friederike Herbst, Tonio J. L. Lang

Supplementary information The online version contains supplementary material available at https://doi.org/10.1038/s41375021-01267-5.

Friederike Herbst

friederike.herbst@nct-heidelberg.de

Extended author information available on the last page of the article steps [5]. Knockout experiments of the miRNA-processing enzymes DGCR8 or DICER in distinct blood populations [6-8] clearly established that miRNAs are crucial for hematopoietic lineage determination.

Up to now, several miRNAs have been identified to play a role in hematopoietic lineage specification $[9,10]$ and stem cell self-renewal [11-13]. As an example, the knockdown of miR-126 in hematopoietic stem cells (HSCs) and early progenitors induces the expansion of the HSC pool without exhaustion by attenuating extrinsic signals [14]. Although more than $50 \%$ of annotated vertebrate miRNAs are intronic and more than two-thirds are co-expressed with their host genes [15], their relationship is not well understood as most studies on miRNA regulation do not address the interplay with their co-regulated genes.

Mechanistically, miRNAs and host genes can interact synergistically or antagonistically [16]. Synergistic functions have been described in cholesterol homeostasis, where miR-33 cooperates with the Sterol Regulatory ElementBinding Protein [17, 18], and neurogenesis, during which 
miR-338 targets genes that are antagonistic to its host the Apoptosis Associated Tyrosine Kinase [19]. By contrast, miR-26b functions in an antagonistic way by directly suppressing the CTD Small Phosphatase 2 [20, 21]. Nevertheless, very little is known about the molecular interaction between a host gene and its miRNA in hematopoiesis.

We and others have previously shown that integration sites (IS) of gamma-retroviral vectors $(\gamma \mathrm{RV})$ into the host cell genome can be used to identify open and active chromatin regions within target cells [22-24]. By systematically analyzing the entire IS repertoire of therapeutic $\gamma \mathrm{RV}$ in ten patients enrolled in the first German Wiskott-Aldrich syndrome (WAS) gene therapy trial we observed that clusters of IS mark hematopoietic regulatory genes, which are active in human bona fide HSCs. Notably, our study supports the application of $\gamma \mathrm{RV}$ derived IS as neutral tags for cell-typespecific regulatory regions and that only a very limited number of $\gamma \mathrm{RV}$ marked loci drive clonal selection [25]. Interestingly, we identified a significant cluster of IS close to the ENAH-VASP-like (EVL)/MIR342 gene locus (among the top 50 tagged gene regions) after thoroughly investigating the IS pattern in peripheral blood cells from nine out of ten efficiently engrafted WAS patients [22, 25]. The continuous clonal contribution of cells harboring $E V L /$ MIR342 vector IS to blood formation for about five years points to a functional role of this genetic region in hematopoiesis. The EVL gene locus has mainly been reported to be involved in filopodia and lamellipodia formation in epithelial cells [26, 27] but has not been linked to hematopoiesis so far. Recently, it has been shown that miR-342 plays a role in the differentiation within the brain [28], but the function of EVL in this context was not investigated. To address the role of this common genomic locus encoding for a protein as well as a miRNA in hematopoiesis, we examined the individual and combinatory influence of EVL and its intronic miR-342 on blood cell differentiation.

\section{Methods}

\section{Generation of lentiviral vectors}

The genetic region of $266 \mathrm{bp}$ harboring the MIR342 locus was amplified from DNA extracted from the murine cell line 32D (for primer sequences see: Supplementary Table S1), subcloned and inserted into the 9928 bp plasmid pFCW.SIN. PPT.UbiC.mCherry.wPRE (LV.mCherry) using the restriction enzymes NheI and AscI, and verified by sequencing.

The codon-optimized coding sequence of the murine $E v l$ gene (NCBI: NM_016337) was synthesized (ThermoFisher Scientific, Invitrogen GeneArt Gene Synthesis, Darmstadt, Germany) and inserted into the 8492 bp plasmid pCCL.SIN. cPPT.PGK.IRES.eGFP.wPRE (LV.eGFP) using the restriction enzymes SbfI and AsiSI, and verified by sequencing. VSV.G-pseudotyped concentrated lentiviral vector stocks for LV.eGFP, LV.Evl, LV.mCherry, and LV. miR-342 were produced by transient co-transfection as described in Herbst et al. [29], with minor modifications. In brief, the co-transfection of lentiviral packaging and transfer vectors was done by complex formation with polyethylenimine (PEI; Sigma, Deisenhofen, Germany) using a DNA:PEI ratio of $1: 3$.

\section{Quantitative real-time PCR analysis and global gene expression profiling}

Total RNA was isolated using the RNeasy Kit (Qiagen, Hilden, Germany) for cell culture cells, or the ARCTURUS $^{\oplus}$ PicoPure ${ }^{\oplus}$ RA Isolation Kit (ThermoFisher Scientific, Applied Biosystems, Darmstadt, Germany) for murine hematopoietic primary cells. For quantitative realtime PCR, total RNA was transcribed in reverse using the Superscript III First-Strand Synthesis System (Invitrogen, Karlsruhe, Germany), and amplified using the Taqman Assay (ThermoFisher Scientific, Dreieich, Germany) or SYBR Green (LC480, Roche Diagnostics, Mannheim, Germany), for primer sequences see Supplementary Tables S2 and S3. The miRNeasy Mini Kit (Qiagen, Hilden, Germany) was used to extract and transcribe miRNA, which were examined by qRT-PCR using the miScript SYBR ${ }^{\oplus}$ Green PCR Kit (Table S3). Signal intensities were normalized against the controls (Rnu6b or Tbp). Eleven days after lentiviral transduction, murine primary $\mathrm{Lin}^{-} \mathrm{Scal}^{+}$ $\mathrm{cKit}^{+}$bone marrow (BM) cells were harvested and the RNA was extracted. Global gene expression profiling was conducted ( $n=2$ biological replicates per group) with the Illumina MouseWG-6v2_BeadChip system at the DKFZ Genomics and Proteomics Core Facility. For further details, see Supplementary Methods. The data discussed in this publication are accessible through GEO Series accession number GSE109600.

\section{Cell culture, lentiviral transduction}

32D cells were cultured in RPMI-1640 (Invitrogen, Darmstadt, Germany) supplemented with $10 \%$ fetal bovine serum (PAN-Biotech, Aidenbach, Germany), 10\% conditioned WEHI-medium, $2 \mathrm{mM}$ L-glutamine (Invitrogen, Darmstadt, Germany) and $1 \%$ penicillin/streptomycin (GibCo, ThermoFisher Scientific, Dreieich, Germany). 293T cells were cultured in Iscove's Modified Dulbecco's Media (Invitrogen, Darmstadt, Germany) supplemented with $10 \%$ FBS, $2 \mathrm{mM}$ L-glutamine, and $1 \%$ penicillin/streptomycin. J77A. 1 cells were cultured in DMEM (4.5 g glucose; Invitrogen, Darmstadt, Germany) supplemented with $10 \%$ FBS, $2 \mathrm{mM}$ L-glutamine, and sodium pyruvate. Jurkat cells were 
cultured in RPMI-1640 supplemented with 10\% FBS and 2 $\mathrm{mM}$ L-glutamine. U-2 OS cells were cultured in McCoy's 5A (GibCo, ThermoFisher Scientific, Dreieich, Germany) supplemented with $10 \%$ FBS and $2 \mathrm{mM}$ L-glutamine.

$\mathrm{Lin}^{-} \mathrm{Scal}^{+} \mathrm{cKit}^{+}(\mathrm{LSK})$ cells were cultured in Stem$\operatorname{Span}^{\mathrm{TM}}$ SFEM (StemCell Technologies, Cologne, Germany) supplemented with $100 \mathrm{ng} / \mathrm{ml} \mathrm{rmSCF}, 100 \mathrm{ng} / \mathrm{ml}$ rmFlt3 Ligand, $100 \mathrm{ng} / \mathrm{ml} \mathrm{rhTPO}, 20 \mathrm{ng} / \mathrm{ml} \mathrm{rmIL} 3$ (all R\&D Systems, Wiesbaden, Germany) and $1 \%$ penicillin/streptomycin. Cell lines were purchased from DSMZ, were authenticated using Multiplex Cell Authentication by Multiplexion (Heidelberg, Germany) as described recently [30], and are routinely tested for mycoplasma contaminations. The lentiviral transduction of 32D, 293T, J774A.1, Jurkat, U-2 OS cells or LSK cells was performed in the presence of Polybrene or Protaminesulfat $(8 \mu \mathrm{g} / \mathrm{ml}$; Sigma, Darmstadt, Germany) using a multiplicity of infection of $1-50$.

\section{Western blot, luciferase assay, and CFC differentiation assay}

Whole-cell extracts were obtained by using a cell lysis buffer (50 mM Tris- $\mathrm{HCl}$ (pH 7.4), $150 \mathrm{mM} \mathrm{NaCl}, 1 \%$ NP40, $0.5 \%$ Na-deoxycholate, $0.1 \%$ SDS, protease inhibitor cocktail). After centrifugation at $13,000 \mathrm{rpm}$ for $20 \mathrm{~min}$, protein lysates were loaded onto SDS-PAGE and transferred to a PVDF membrane (Invitrogen, Darmstadt, Germany). Antibodies are listed in Supplementary Table S4.

For luciferase assays, $1-5 \times 10^{4} 293 \mathrm{~T}$ cells either transfected with $0.5 \mu \mathrm{g}$ miR-342 expression construct, $0.02 \mathrm{ng}$ miR-342-3p or miR-342-5p mimics (miRIDIAN, Horizon Discovery, Cambridge, UK) or 293T cells ectopically expressing miRNA or control vector were used. 293T cells were further transfected using PEI with 0.05 or $0.25 \mu \mathrm{g}$ psiCheck2 vectors (Promega, Walldorf, Germany), respectively containing the target gene-specific $3^{\prime} \mathrm{UTR}$ - or miR342-binding sites of the sponge vector (cloning primers in Table S1). After $48-72 \mathrm{~h}$ of transfection, the luciferase activity was measured using the Dual-Luciferase ${ }^{\circledR}$ Reporter Assay System Kit (Promega, Walldorf, Germany) according to the manufacturer's protocol. All experiments were performed in biological replicates $(n=3)$ using technical replicates $(n=3)$. Investigators were blinded to group allocation until the evaluation of data followed by interpretation.

For myeloid colony-forming unit (CFU) assays, 4000 fluorescence-positive LSK cells were plated using MethoCult $^{\mathrm{TM}}$ GF M3434 (StemCell Technologies, Cologne, Germany). For lymphoid CFU assays, 8000 sorted LSK cells were plated using Methocult ${ }^{\mathrm{TM}} \mathrm{M} 3630$. Colonies were quantified 10 days after incubation at $37^{\circ} \mathrm{C}$ and $5 \% \mathrm{CO}_{2}$. All CFU assays were performed in biological replicates $(n=3)$ using technical replicates $(n=2)$.

\section{AG02 co-immunoprecipitation, low RNA high- throughput sequencing, and bioinformatical analysis}

A total of $2 \times 10^{7}$ cells per sample were lysed with an ice-cold polysome buffer (10 mM HEPES (pH 7), $100 \mathrm{mM} \mathrm{KCl}$, $5 \mathrm{mM} \mathrm{MgCl}_{2}, 0.5 \%$ NP-40, $10 \mathrm{mM}$ DTT, 2.25 U RNaseOut, protease inhibitor cocktail, $0.4 \mathrm{mM}$ Vanadyl ribonucleoside complex), and stored overnight at $-80^{\circ} \mathrm{C}$. Immunoprecipitation was performed with $5 \mu \mathrm{g}$ of anti-Ago 2 antibody, or the mouse $\operatorname{IgG}$ isotype control coupled with Dynabeads (ThermoFisher Scientific, Dreieich, Germany) for $4 \mathrm{~h}$ at $4{ }^{\circ} \mathrm{C}$ in NT2 buffer (50 mM Tris (pH 7.4), $150 \mathrm{mM} \mathrm{NaCl}, 1 \mathrm{mM}$ $\mathrm{MgCl}_{2}, 0.05 \% \mathrm{NP}-40,0.5 \mathrm{U}$ RNaseOut, $0.4 \mathrm{mM}$ Vanadyl ribonucleoside complex, $1 \mathrm{mM}$ DTT, $0.015 \mathrm{mM}$ EDTA $(\mathrm{pH} 8)$, protease inhibitor). After washing the beads three times with ice-cold NT2 buffer, the proteins were eluted by incubating the samples with Glycin ( $\mathrm{pH} \mathrm{2.3)}$ ) for $15 \mathrm{~min}$ followed by neutralization with Tris- $\mathrm{HCl}(\mathrm{pH} 8)$. Proteins were digested using Proteinase $\mathrm{K}$ and removed by QIAzol/ Chloroform extraction. RNA was isolated using the miRNeasy Kit according to the manufacturer's protocol.

Total RNA fractions ( $n=2$ technical replicates/group) were submitted for low RNA HTS using Illumina HiSeq2000 (paired end, 125 bp). For further details see Supplementary Methods. The common predicted miR-342 target genes (from miRWalk, miRanda, RNA22, and Targetscan) were downloaded from miRWalk2.0 and used in BioVenn to identify the intersection between genes expressed in LSK cells (RPKM $>800)$ and the enriched genes after $\mathrm{AGO} 2$ pulldown.

\section{Flow cytometry}

Cells (for details about the immunophenotype of enriched cell fractions see Table S5) were dissociated, washed once with Hanks' balanced salt solution (HBSS; Sigma, Darmstadt, Germany) containing 2\% FBS, and analyzed for eGFP or mCherry positivity by flow cytometry (AriaII, LSRII, FACS-Fortessa; Becton Dickinson, Heidelberg, Germany).

The peripheral blood from the recipient mice was collected monthly. After lysis of erythrocytes $(0.15 \mathrm{M}$ ammonium chloride), the cells were washed with HBSS containing 2\% FBS, stained with Fluoro-Gold (ThermoFisher Scientific, Dreieich, Germany) for dead cell exclusion and with fluorochrome-conjugated monoclonal antibodies (Table S6), for further analysis by FACS for eGFP or mCherry expression in addition to lineage markers. Spleens were harvested, cells were singularized using a cell strainer $\left(40 \mu \mathrm{m}\right.$, Falcon $\left.{ }^{\circ}\right)$, washed and stained for hematopoietic lineage markers. 
Bone marrow transplantation and colony-forming unit-spleen

BM cells were harvested from the hind legs and spines of 6-8-week-old male B6.SJL-Ptprc ${ }^{\mathrm{a}} \mathrm{Pepc}^{\mathrm{b}} /$ BoyJ (Ly 5.1) mice, transduced with $\mathrm{LV}$, and injected intravenously into lethally irradiated (950 cGy) female C57B16/J (Ly 5.2) mice [29]. In brief, lineage depletion of the harvested BM was performed using EasySep (StemCell Technologies, Cologne, Germany), further enriched for LSK markers (Table S6) by FACS, and transduced overnight with LV. After $36 \mathrm{~h}$, cells were injected intravenously into lethally irradiated recipients (2500 LSK cells per mouse, $n=6$ per group). BM and spleens were harvested after 4 months and $2 \times 10^{7}$ total $\mathrm{BM}$ cells/mouse were re-transplanted into secondary recipients ( $n=6$ /group).

For CFU-Spleen limiting-dilution assays, 530, 1440, or 14,400 cells per mouse were injected intravenously into lethally irradiated recipients ( $1050 \mathrm{cGy})$. After 13 days of transplantation, spleens were harvested, weighed, and colonies were counted. Investigators were blinded to group allocation until mice were sacrificed. L-Calc Software was used to determine the frequency of CFU-S. $5(+1$ substitute) mice were used per group as explorative cohort and randomly allocated into experimental groups. Mice were housed under pathogen-free conditions and all experiments were conducted according to the German animal protection laws and regulations approved by the ethical committee.

\section{Statistical analysis}

Results are presented as mean \pm s.d. if not indicated differently. Statistical significance was determined using the Student's $t$-test. Biological triplicates and technical replicates were used, if not indicated differently.

\section{Results}

\section{IS-tagged EVL/MIR342 hematopoietic clones are long-term active in human hematopoiesis}

We identified the EVL/MIR342 locus by subsequently analyzing the whole integrome of ten patients treated with clinical gene therapy for the correction of WAS for up to 6 years post transplantation [22, 25]. WAS is an X-linked, primary immunodeficiency disorder caused by mutations in the WAS gene. Patients suffer from recurrent infections, thrombocytopenia, and autoimmunity, and have an increased risk of lymphoma development [31]. The mutated WAS protein leads to dysfunctional leukocytes including lymphoid and myeloid cells [32]. Treatment options consist of allogeneic HSC transplantation or gene therapy approaches using patient-derived $\mathrm{CD} 34^{+}$cells for genetic modification. Here, we discuss data from the German WAS gene therapy trial [22] using $\gamma \mathrm{RV}$ gene delivery vehicles.

Hematopoietic clones carrying therapeutic vector integrations near the EVL/MIR342 locus were detected in peripheral blood and BM samples of all patients post-transplant and this locus was among the top 50 enriched regions marked by common integration sites (CIS). Recently, we showed that the number of CIS in hematopoietic clones of WAS patients strongly correlates with chromatin accessibility in HSCs but not with the level of gene expression of the IS-tagged loci [25]. As gamma-retrovirus derived vectors can serve as neutral molecular tags by preferentially integrating into cell-type-specific active and open chromatin regions [24, 33, 34], we hypothesized that the $E V L$ and MIR342 genes are expressed and functionally relevant in normal human long-term hematopoiesis. Moreover, clonal outgrowth due to insertional mutagenesis is restricted to a very defined and limited number of clones and there is no known function of $E V L$ and MIR342 genes in WAS.

First, we analyzed the genomic elements close to the 165 insertions derived from unique clones (identified in all patients analyzed) in vicinity to the EVL/MIR342 locus. Vector integrations within patient-derived cells clustered close to the transcription start site (TSS) of EVL (+44.4 to $-75.5 \mathrm{~kb}$ ) (Fig. 1a). Next, we applied the same selection criteria to conserved miRNA genes only and identified miR342 as the second top-ranked miRNA following miR-132/ 212 (Fig. 1b), a known regulator of HSC maintenance [13]. Eight out of the top ten-ranked miRNAs have been proven to play a role in hematopoiesis. Furthermore, $40 \%$ of the unique clones were detected more than once within individual patients (Fig. 1c) and 21 clones out of those were sequenced several times for the observation period of more than 5 years in hematopoietic samples clearly showing their long-term hematopoietic activity (Fig. 1d).

\section{Evl and miR-342 are co-expressed in murine hematopoietic cells}

We analyzed the expression of EVL in human blood cell populations starting from HSCs via progenitors to differentiated cells (Fig. 1e) using publicly available datasets from Corces et al. (https://doi.org/10.1038/ng.3646) and next verified the expression level of Evl and miR-342-3p in defined murine hematopoietic cell populations (Fig. 1f, g). It is important to note that the conservation of the EVL protein is $94 \%$ between human and mouse (Supplementary Fig. S1a) and that both species share $100 \%$ identical $5 p$ and $3 p$ mature miR-342 sequences.

Both genes were expressed at low level in murine HSC and progenitor cells (MPP1-4), which is in line with published whole-transcriptome sequencing data [35], and their 
a.

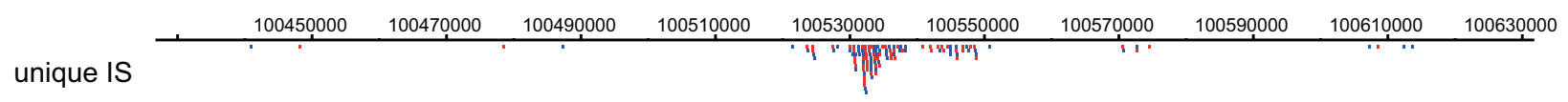

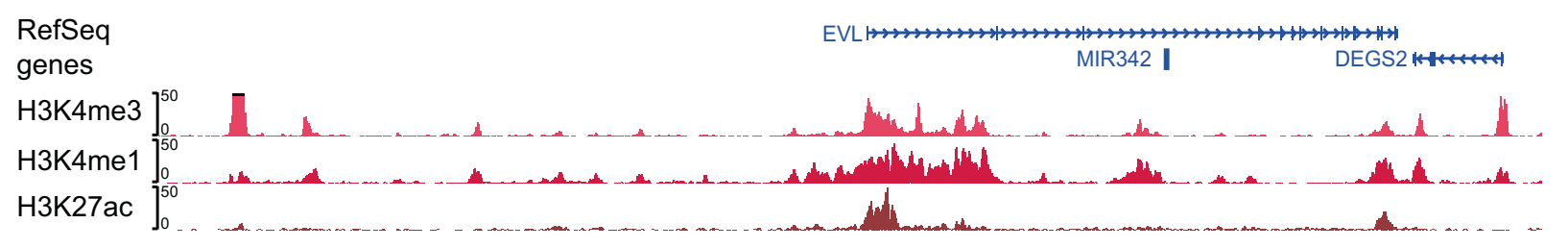

b.

\begin{tabular}{|c|c|c|}
\hline rank & miRNA & \#ISs \\
\hline 1 & miR-132/212 & 229 \\
\hline 2 & miR-342 & 162 \\
\hline 3 & miR-10a & 91 \\
\hline 4 & miR-326 & 78 \\
\hline 5 & miR-148b & 73 \\
\hline 6 & miR-221 & 70 \\
\hline 7 & miR-142 & 67 \\
\hline 8 & miR-146a & 66 \\
\hline 9 & miR-17 & 64 \\
\hline 10 & miR-223 & 59 \\
\hline
\end{tabular}

e.

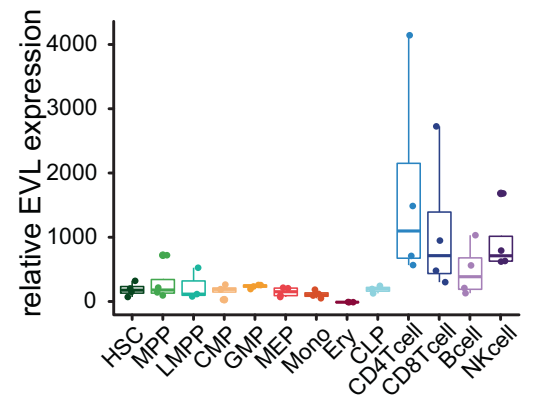

Fig. 1 Identification of the $E V L / M I R 342$ gene locus as a novel candidate regulatory region in hematopoiesis. a Genomic localization of all therapeutic vector integration sites (IS) close to the $E V L /$ MIR342 gene locus detected in ten Wiskott-Aldrich syndrome gene therapy patients. Histone modifications in human $\mathrm{CD} 34^{+}$cells representing promoter (H3K4me3), enhancer (H3K4me1) and active (H3K27ac) chromatin regions. H histone, K Lysine, me methylated, ac acetylated. b Top-ranked miRNAs according to the number of therapeutic vector integration sites (ISs) nearby in efficiently engrafted Wiskott-Aldrich syndrome patients $(n=9)$. c Percentage of IS, which were sequenced once (light red), more than once (red) and more than twice (dark red) in WAS gene therapy patients during the cause of the study (2097 days post-transplant). d Clonal dynamics of individual gene-corrected cells harboring IS close to the EVL/MIR342 locus,

expression increased profoundly in differentiated blood cells (Fig. 1f, g). The highest expression of Evl was detected in lymphocytes (13.8 $\pm 0.3-19.1 \pm 0.9$-fold higher compared to MPP4 cells), whereas miR-342 was expressed at the highest level in macrophages $(85.8 \pm 0.4$-fold higher compared to MPP4 cells).

However, we observed co-expression of both gene products in murine hematopoietic primary cells (Fig. 1f, g) as d.

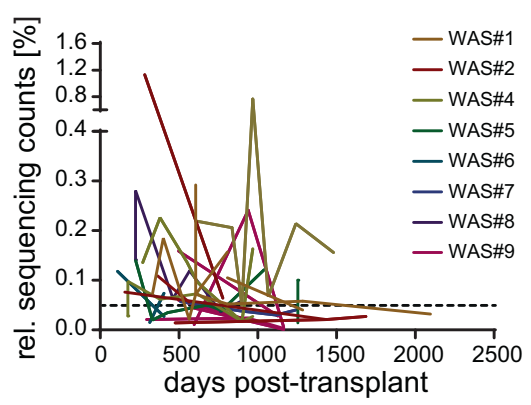

g.

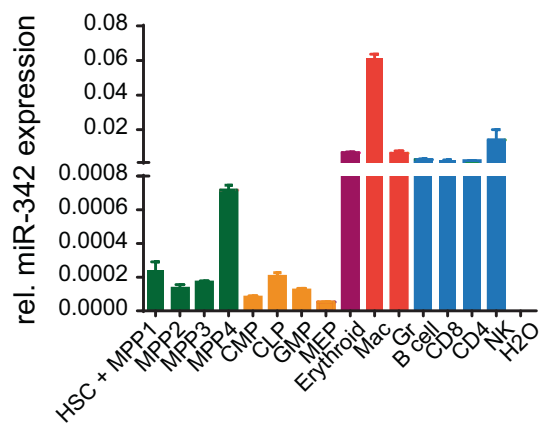

which were repeatedly (>twice, Fig. 1b) detected in WAS patients for up to 2097 days post-transplant. Dashed line represents the median of all repeatedly sequenced IS. e RNA-seq counts for EVL transcripts in defined human hematopoietic cell populations. f Relative expression of Evl (normalized to Tbp) and $\mathbf{g}$ miR-342-3p (normalized to Rnu6b) in enriched murine hematopoietic primary populations. Indicated cell populations were isolated from 8- to 10-week-old C57/B16 mice using immunophenotypic markers, RNA was isolated and subjected to qRTPCR. HSC hematopoietic stem cell, MPP multipotent progenitor, CMP common myeloid progenitor, CLP common lymphoid progenitor, GMP granulocyte macrophage progenitor, MEP megakaryocytic erythroide progenitor, Gr granulocyte, Mac macrophage, CD8 T cell, CD4 T cell, NK1.1 natural killer cell, n.d. no data.

well as in murine and human cell lines (Supplementary Fig. $\mathrm{S} 1 \mathrm{~b}, \mathrm{c})$. This suggests that the intronic miR-342 is coregulated with its host gene $E v l$, which is in line with its previous classification as a canonical and host gene coexpressed miRNA [16, 28]. To gain insights into the regulatory landscape and chromatin structure of the locus in human hematopoietic cells, we analyzed a recently published data set from Corces et al. defining open chromatin 
by ATAC-Seq (Assay for Transposase Accessible Chromatin with high-throughput sequencing) in human CD34 cells and physical genetic interaction determined by promoter capture $\mathrm{HiC}$ sequencing published by Mifsud et al. (https://doi.org/10.1038/ng.3286). We identified enhancer marks (H3K4me1) upstream of both, the EVL TSS and the MIR342 gene locus (Fig. 1a). Yet, acetylated H3K27 indicating active chromatin was only detected upstream of $E V L$. Genomic interaction was indicated between the MIR342 region and the promoter/enhancer sequences in front of the EVL TSS (Fig. S2), and myeloid transcription factor binding sites (data not shown) were present close to MIR342, which is in accordance to its highest expression in murine myeloid cells (Fig. 1g). Noteworthy, the chromatin accessibility at the MIR342 locus was lowest in human common lymphoid progenitors (CLPs) and B-cells compared to all other cell types (Fig. S2).

\section{Overexpression of EVL drives lymphopoiesis whereas miR-342 promotes myeloid differentiation}

Firstly, we validated the stable lentiviral mediated overexpression (OE), which is comparable with physiologic levels detected in murine and human cell lines or hematopoietic primary cell populations (data not shown), and assessed the functionality of Evl and miR-342-3p in 32D and 293T cells (Fig. S3). In a next step, we addressed whether OE of either Evl or miR-342 affects the differentiation capacity of murine stem and progenitor LSK cells in vitro and in vivo (Fig. 2a). OE of Evl led to a 7.5 \pm 5.1 fold increase of pre-B cell colonies compared to control vector-transduced LSK cells (Fig. 2b). By contrast, miR-342 overexpressing cells formed a $2.3 \pm 0.6$-fold higher number of myeloid colonies (Fig. 2c). To quantify the myeloid colony-forming potential of miR-342 ${ }^{+}$LSK cells in vivo, we analyzed myeloid spleen colonies after transplantation. In line with the in vitro data, we detected enlarged spleens (Fig. 2d; group \#1, $P=0.047$ ) and a $2.4 \pm 0.6$-fold increase in colonies (Fig. 2e; $P=0.0183$ ) due to a higher number of miR-342-positive donor-derived cells (Fig. 2f, $P=0.007$ ). In total, we assessed a frequency of $1 / 275$ CFU-S in miR$342^{+}$LSK cells compared to $1 / 486$ CFU-S in control vectortransduced LSK cells determined by limiting dilution, as well as a higher proportion of donor-derived myeloid cells in the spleens (Fig. $2 \mathrm{~g} ; P=0.042$ ). Serial BM transplantation experiments demonstrated that both, EVL and miR-342 overexpressing cells, generated stable multilineage reconstitution (Fig. 2h-k). However, a 4.3-fold higher frequency of EVL-positive B-cells was detected in peripheral blood 4 and 8 weeks $(P=0.93 \mathrm{e}-6)$ after secondary transplantation (Fig. 2i). Moreover, $\mathrm{EVL}^{+}$primary and secondary recipients harbored a higher donor-derived B-cell frequency within their spleen $\left(1^{\circ}\right.$ spleen: $P=0.06$ and $2^{\circ}$ spleen: $P=0.07$;
Fig. S4) but donor-derived granulopoietic cell numbers were largely unaffected (Fig. 2k).

To gain further insights into the molecular function of EVL and miR-342, we performed global gene expression profiling of transduced LSK cells. We observed 191 and 85 significantly differentially expressed genes upon Evl or miR-342 OE, respectively (Fig. 3a). Interestingly, 32\% (62 out of 191) of the deregulated transcripts were involved in hematopoietic system development and function after Evl OE. Moreover, the top deregulated canonical pathways detected are essential for the development of B-cells $(P=$ $0.26 \mathrm{e}-12)$. By contrast, genes relevant to myeloid cell functions, such as granulocytic adhesion and diapedesis $(P$ $=0.26 \mathrm{e}-4$ ) were significantly upregulated within miR-342positive LSK cells (Fig. 3b). Next, we used the upstream regulator analysis to identify the activity of gene regulatory networks, and to predict transcriptional regulators. The activation z-score was used to select the top ten activated and inhibited pathways upon Evl OE. Here, we revealed that apoptosis and cell death-associated pathways are inhibited, whereas lymphopoiesis, cell movement and invasion are activated (Fig. S3e). In particular, interferon and cytokine signaling, MHC class II antigen presentation and TCR activation are enriched indicating for pathways in developing lymphocytic cells (data not shown). Subsequently, we extracted the top 20 predicted cellular upstream regulators identified based on the $\mathrm{Evl}^{+}$LSK cell transcriptome (Fig. 3c). As an example, we found TRAF6 mediated NFKB signaling activated in hematopoietic primary cells upon Evl OE (z-score $3.2 ; p$ value of overlap genes $P=0.28 \mathrm{e}-11$, Fisher's exact test). To identify drivers of lymphoid diversification, we selected for deregulated transcription factors in $\mathrm{EVL}^{+} \mathrm{LSK}$ cells compared to mock transduced cells (Fig. 3d). Egr2, with the highest log2 FC of 1.8, has been shown to regulate Hox genes like HoxA4, important for HSC and proB cell expansion [36] or HoxB 3 blocking $\mathrm{B}$ cell progenitors, $\mathrm{T}$ cell differentiation or delaying myeloid precursor proliferation [37] when overexpressed which suggests for a role in balancing myeloid and lymphoid fractions. Thus, EVL is associated with drivers of lymphopoiesis, whereas miR-342 OE results in upregulation of myeloid-specific pathways.

\section{Antagonism of miR-342 and EVL in hematopoietic lineage differentiation}

As miR-342 is co-expressed with its host gene $E v l$, we analyzed hematopoietic differentiation after simultaneous $\mathrm{OE}$ of both genes. Interestingly, miR-342 expression in $\mathrm{EVL}^{+}$LSK cells reduced the lymphoid-primed differentiation capacity of EVL $0.43 \pm 0.29$-fold (Fig. 4a). Furthermore, co-expression of Evl and miR-342 abolished the myeloid-biased colony formation induced by miR-342 OE 
a.
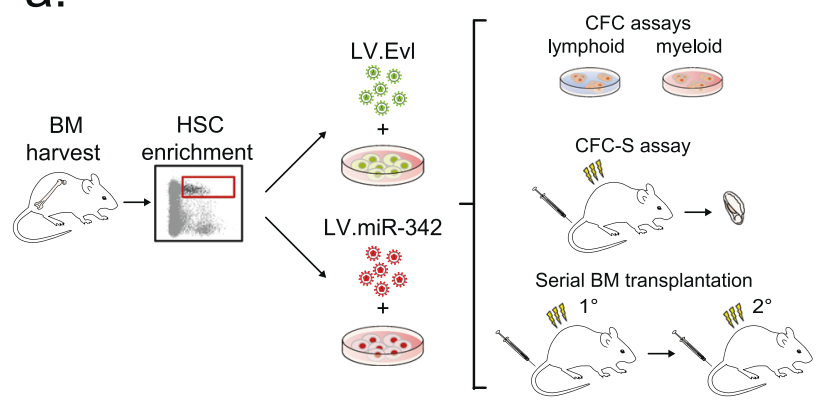

d.
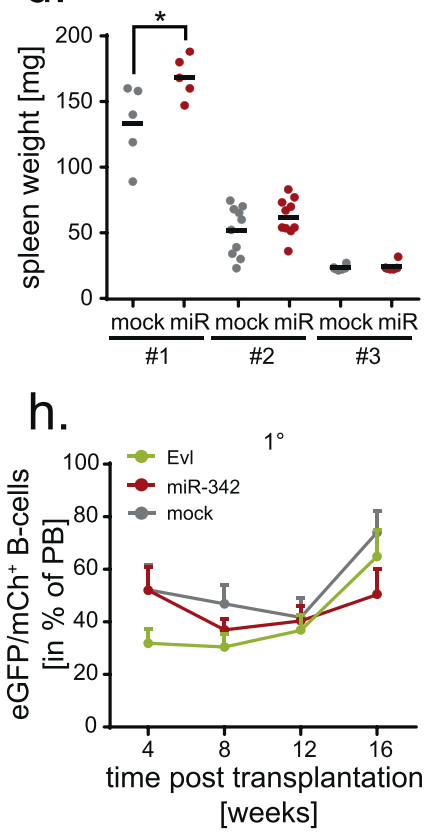

e.
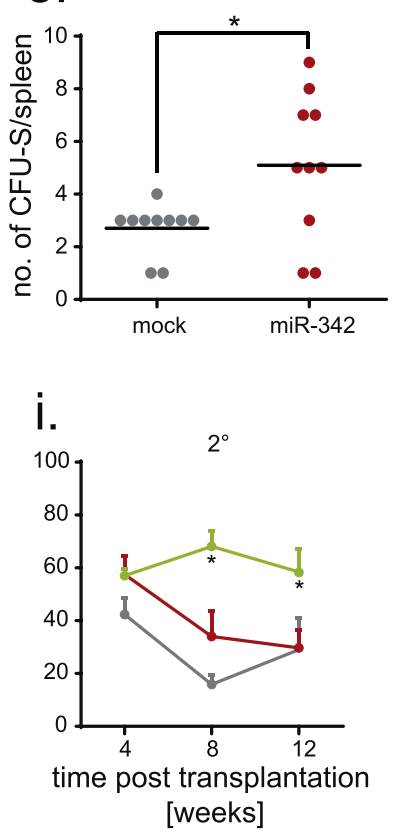

Fig. 2 EVL drives lymphopoiesis whereas miR-342 promotes myeloid differentiation in vitro and in vivo. a Experimental setup scheme. Bone marrow (BM) was harvested from B16 mice, enriched for $\mathrm{Lin}^{-} \mathrm{Scal}^{+} \mathrm{cKit}^{+}$(LSK) cell markers by flow cytometry and cells were transduced with overexpression $(\mathrm{OE})$ lentiviral vectors for Evl or miR-342 (and mock as a control). The influence of Evl and miR-342 $\mathrm{OE}$ on the hematopoietic differentiation capacity was assessed by lymphoid and myeloid colony-forming assays (CFC) in vitro or a CFC-Spleen (S) assay in vivo. Multilineage reconstitution and selfrenewal were addressed by serial $\mathrm{BM}$ transplantation. b Relative number of colonies derived from EVL-, miR-342- or mock overexpressing LSK cells in pre-B-cell growth supporting semisolid medium $(n=3)$. c Relative number of colonies derived from EVL-, miR-342- or mock overexpressing LSK cells in erythroid and myeloid growth supporting semisolid medium $(n=3)$. d Weight of spleens in mg of mice transplanted with 14,400 LSK cells/mouse (group \#1),

alone (Fig. 4b). In vivo, the frequency of donor-derived Tcells detected in the spleen of secondary mice was reduced upon miR-342 OE compared to Evl OE or controls (Fig. S4b).

We performed in silico analysis to identify miR-342 targets $(3 p$ and $5 p)$ and selected 1214 potential candidates (Fig. 4c). Strikingly, miR-342 predominantly targets pathways involved in cell movement (especially T-cell b.

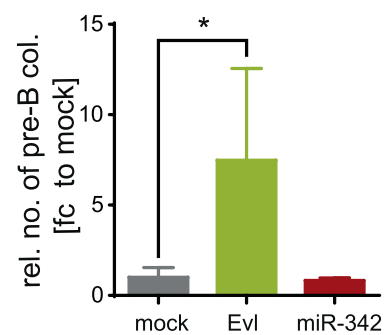

f.

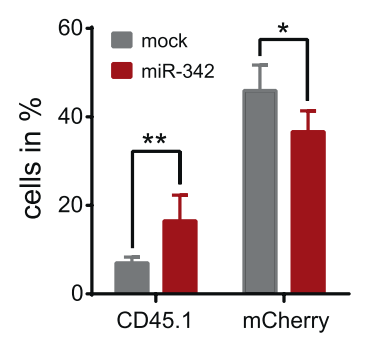

j.

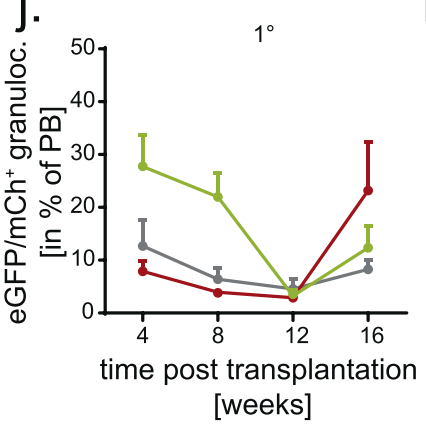

k.

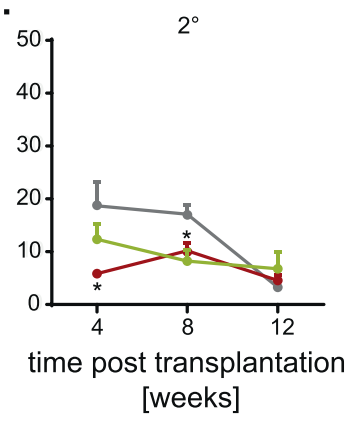

1440 LSK cells/mouse (group \#2), and 530 LSK cells/mouse (group \#3) transduced with miR-342 or mock overexpression vectors 13 days post-transplant. e Number of myelopoietic progenitor-derived colonyforming units (CFU) within the spleens of mice transplanted with LSK cells OE miR-342 or mCherry control 13 days post-transplant. f Frequency of donor-derived (CD45.1) and transduced (mCherry) cells within spleens of mice at day 13 after transplantation of miR-342 OE or mock LSK cells. g Frequency of myeloid (CD11b, Gr1) and lymphoid (CD3, CD45R) hematopoietic cells within the mCherry-positive donor cell fraction in spleens of mice at day 13 after transplantation of miR-342 OE or mock LSK cells. h Frequency of donor-derived eGFP/ mCherry-positive peripheral B-cells in primary $\left(1^{\circ}\right)$ and $\mathbf{i}$ secondary $\left(2^{\circ}\right)$ recipient mice. j Frequency of donor-derived eGFP/mCherrypositive peripheral granulocytes in primary $\left(1^{\circ}\right)$ and $\mathbf{k}$ secondary $\left(2^{\circ}\right)$ recipient mice. $* P<0.05 ; * * P<0.01 ; * * * P<0.001 ; \mathbf{h}-\mathrm{k}(n=6$ mice/ group; SEM).

migration as major subcategory), differentiation and proliferation (main cell class affected: lymphocytes; not shown) (Fig. 4d). This suggests that miR-342 antagonizes its host gene's function, which drives lymphopoiesis. It is important to note that the Evl mRNA does not contain any miR-342 binding sites and that we observed no significant influence on Evl mRNA levels upon ectopic miR-342 expression and vice versa (Fig. S5a, b), pointing towards an indirect and 
a.

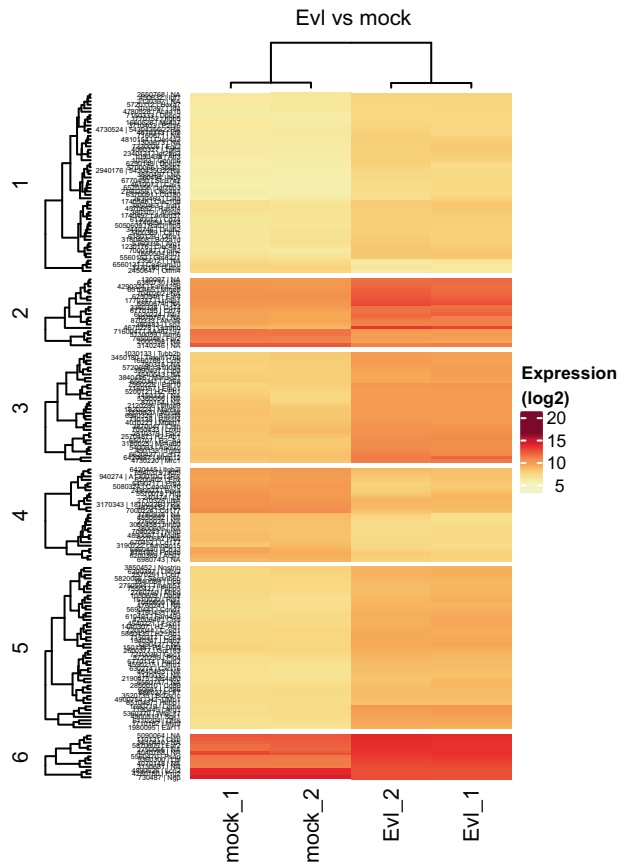

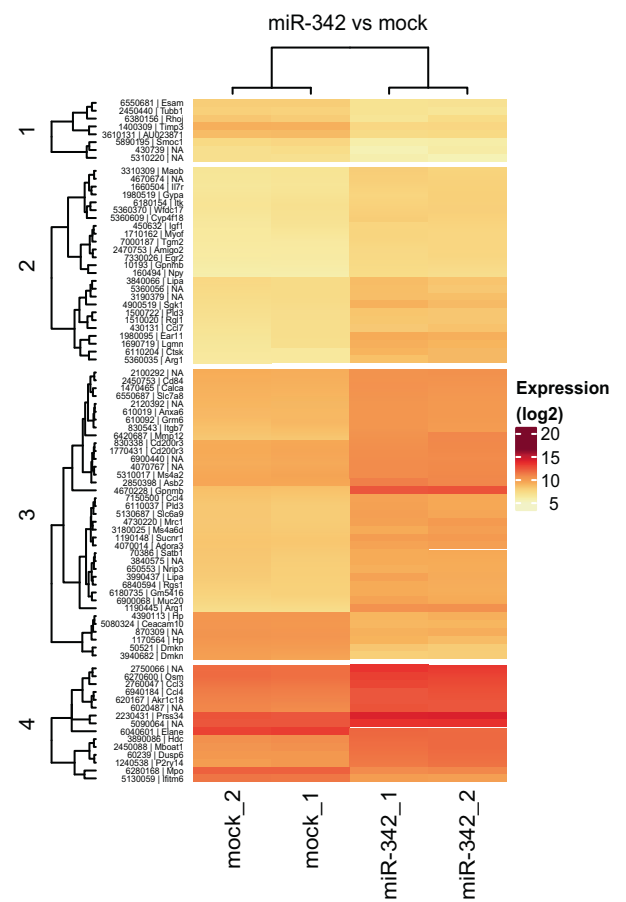

b.

Pathways enriched in EVL positive LSK cells

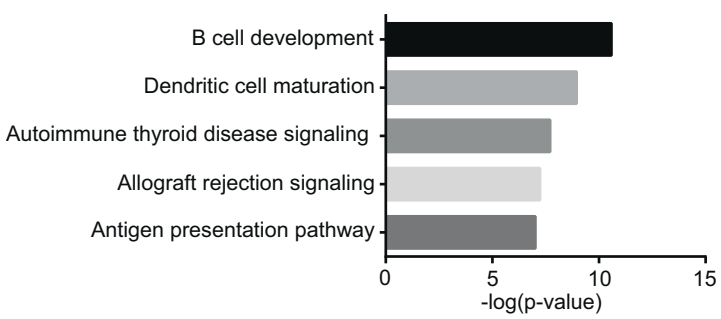

C.

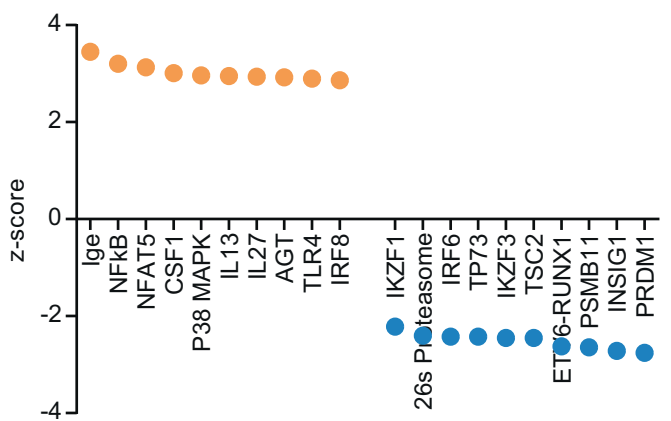

Fig. 3 Overexpression of Evl deregulates pathways important to lymphoid cells whereas miR-342 alters myeloid associated signaling. a Global gene expression profiling of LSK cells overexpressing Evl or miR-342 after 11 days in culture. 190 and 90 transcripts were significantly deregulated (FoldChange $>2$ ) in Evl or miR-342 overexpressing cells compared to mock transduced cells, respectively. b The top five deregulated pathways upon Evl or miR-342 overexpression are displayed ranked by their $-\log (p$ value $)$. c Potential
Pathways enriched in miR-342 positive LSK cells

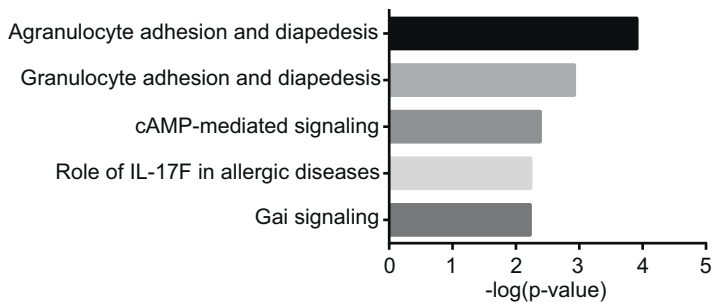

d.

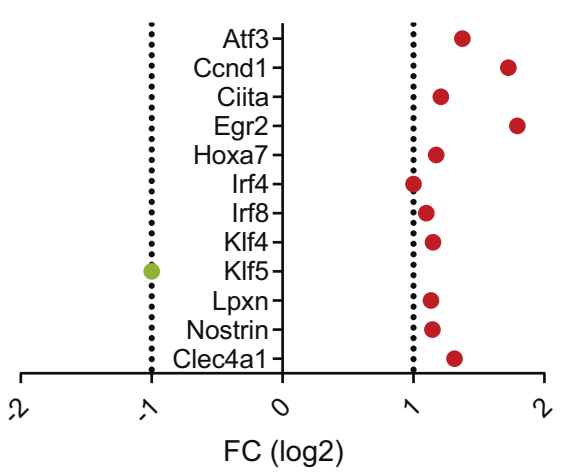

upstream regulators identified in primary LSK cells after overexpression of Evl. Orange circles indicate activation (positive z-score) in murine primary LSK cells upon Evl expression and blue circles represent potential inhibited regulators (negative z-score) based on microarray data analysis using the Ingenuity Pathway Analyzer in Evl expressing (Evl OE) and mock transduced control (ctrl) LSK cells. d FC in $\log 2$ of the 12 transcription factors deregulated in Evl OE LSK cells compared to mock ctrl. 
a.

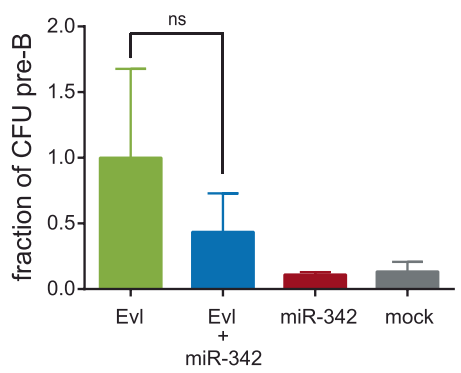

d.

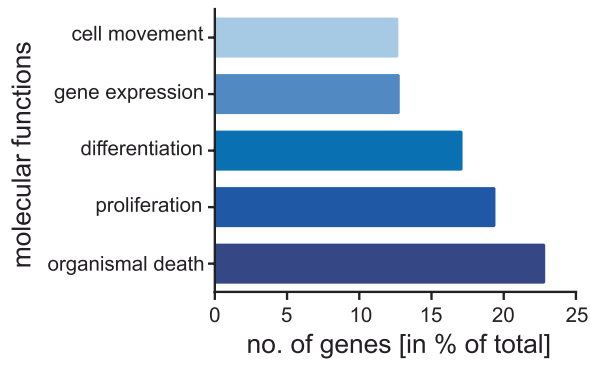

b.

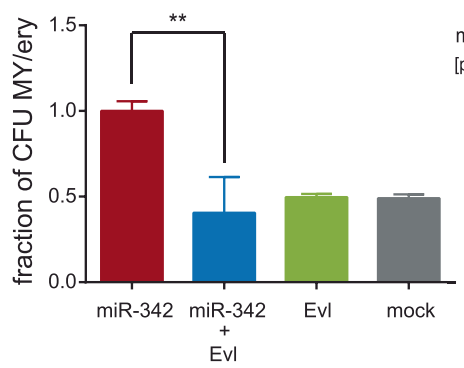

e.

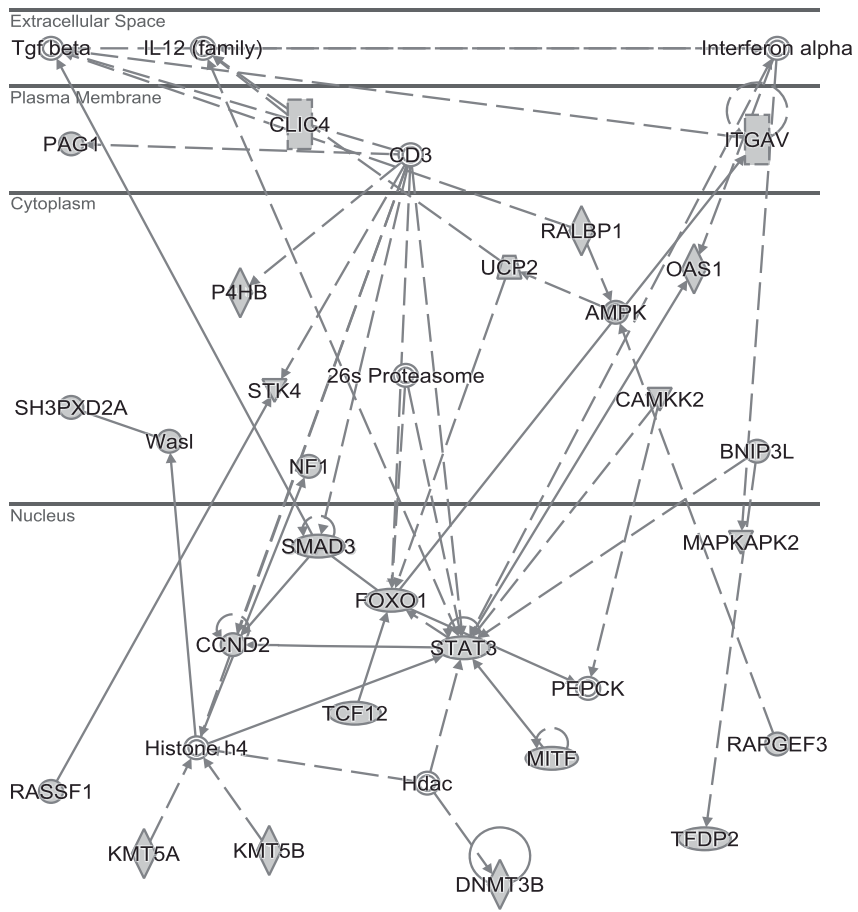

C.

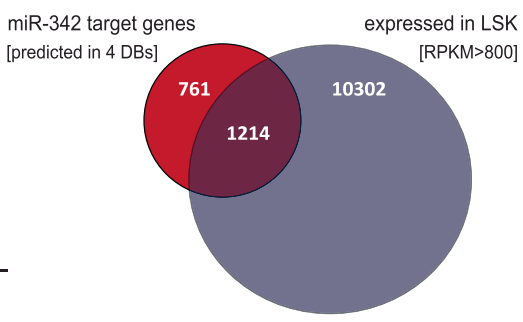

Fig. 4 Co-expression of the host gene Evl and its intronic miR-342 neutralizes their individual hematopoietic differentiation potential. a Relative fraction of colony-forming units (CFU-pre B) derived from LSK cells in pre-B cell growth supporting semisolid medium upon Evl, Evl and miR-342, miR-342 or mock transduction. b Relative fraction of colony-forming units (CFU-GM) derived from LSK cells in myeloid and erythroid (My/ery) growth supporting semisolid medium upon miR-342, miR-342 and Evl, Evl or mock transduction. c Predicted miR-342 target genes identified in four different databases (DB: miRWalk, miRanda, RNA22, and TargetScan) and their expression on
RNA level $($ RPKM $>800)$ in murine primary LSK cell populations [35]. d Pathway analysis using the 1214 in silico-derived miR-342 target genes (Fig. 4c) to describe their molecular functions ranked by the number (no.) of genes in $\%$ of total genes within the given category. e One of the top three networks (Score 30-36) targeted by miR342 identified by in silico analysis (adapted from Ingenuity Pathway Analysis). All identified miR-342 targets within this network are displayed with gray shaded symbols. Solid lines represent direct and dashed lines indirect interactions between molecules based on the IPA database. downstream mode of action. One of the top miR-342 inhibited networks in silico is downstream of CD3, which is part of the T-cell receptor (Fig. 4e). To confirm miR-342 target genes experimentally, we performed AGO2 coimmunoprecipitation and detected a strong enrichment (8674-fold compared to $\mathrm{IgG}$ ) of the ectopic miR-342 in the RISC complex without replacing and unphysiologically outcompeting endogenously expressed miRNAs like miR21 and miR-142 (Fig. 5a). High-throughput sequencing of AGO2 co-immunoprecipitated mRNA fractions revealed 1327 significantly enriched mRNAs ( $\geq 1.5$-fold compared to
AGO2-IP samples of mock cells), of which $73.32 \%$ were bound with their $3^{\prime}$ UTR. Next, we identified 99 candidates whose mRNA expression in LSK cells overlaps with the prediction and experimental assessment as a miR-342 target (Fig. 5b; Table S7). To validate the experimental strategy, we selected seven genes with different mRNA enrichment scores (fold changes between 3.0 and 25.7) in the miR-342versus control-immunoprecipitated AGO2-complexes. We assessed the miR-342 dependent repression of gene-specific 3'UTR containing luciferase constructs (Fig. 5c, Fig. S6) and showed that increasing inhibition of miR-342 leads to 
a.

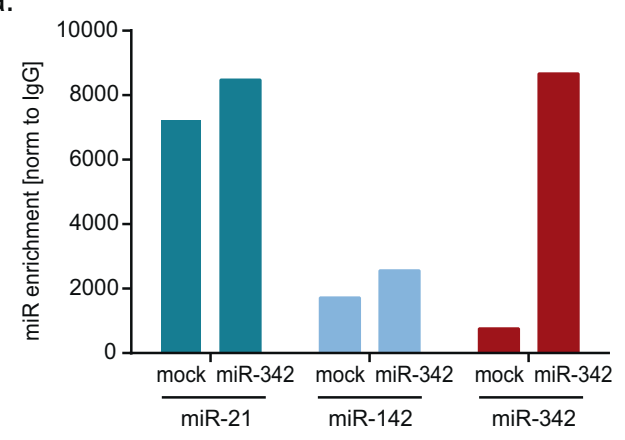

C.

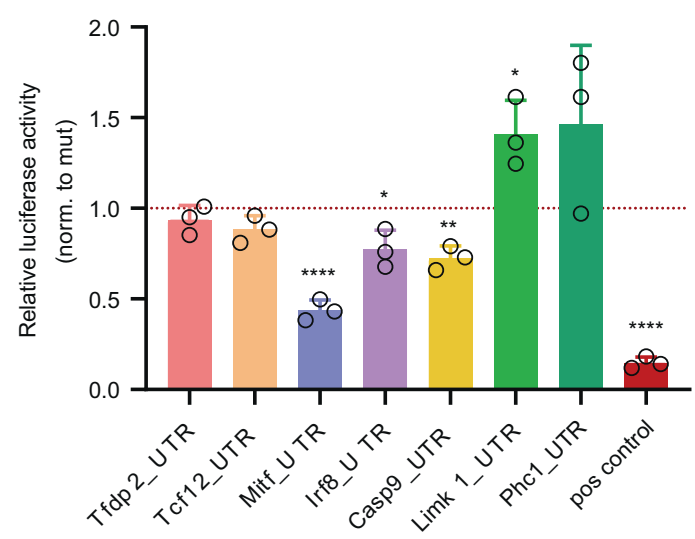

e.

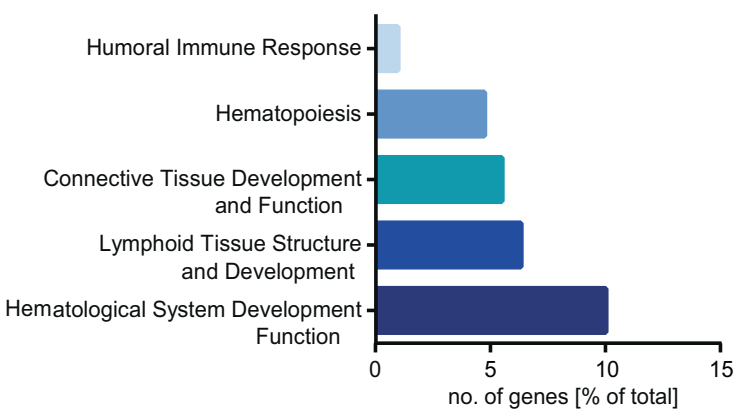

Fig. 5 MiR-342 antagonizes EVL by targeting mRNAs, which are involved in lymphoid signaling pathways. a Relative enrichment of the indicated miRNAs (miR-21, miR-142, miR-342) in mock and miR-342 (miR) overexpressing hematopoietic cells after AGO2 immunoprecipitation compared to IgG control samples. b Venn diagram of experimentally identified miR-342 target mRNAs (AGO2-IP enriched mRNAs) compared to in silico predicted targets and mRNAs expressed in LSK cells (Fig. 4c). c Relative luciferase activity (normalized to individual mutated UTR vector-transfected cells) in 293 T cells after transfection with specific $3^{\prime}$ UTR sequence containing

gradually elevated protein levels of four in silico predicted and AGO2-IP detected miR-342 targets (Fig. 5d), which are suggested to be involved in lymphoid signaling pathways b.

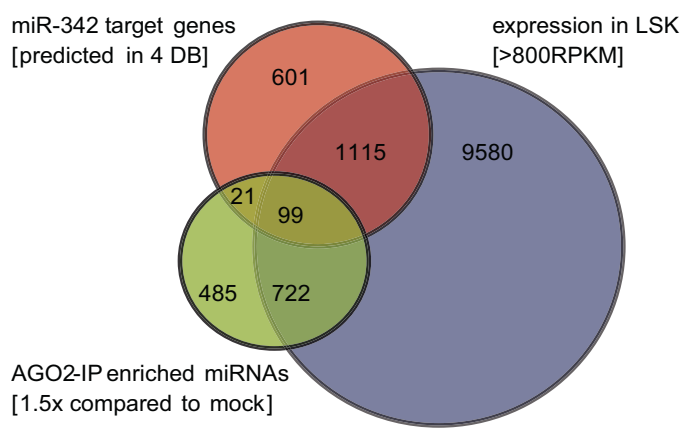

d.

LV.miR-342-sponge LV.GFP-control

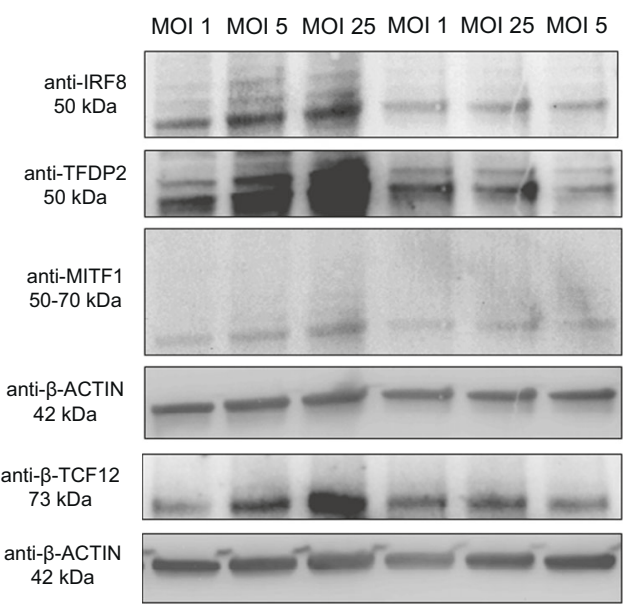

constructs of predicted miR-342 target genes and the miR-342 overexpression vector. $* P<0.05 ; * * P<0.01 ; * * * P<0.001$. d Western blot analysis of identified miR-342 target genes with lysates of murine bone marrow-derived 32D cells after inhibition of miR-342 using increasing amounts (MOI 1, 5, 25) of a specific miR-342 sponge vector (LV.miR-342-sponge) or its corresponding GFP-expressing control vector (LV.GFP-control). e Functional classification of the mRNAs, which are 1.5-fold enriched in miR-342 overexpressing samples compared to mock cells after AGO2-IP ranked by the number (no.) of genes in $\%$ of total genes within the given category.

(see Fig. 4e). Among the top canonical pathways suppressed by miR-342 are SAPK/JNK signaling and B-cell activating factor signaling, which belong to the molecular 
categories of hematological system development and function and lymphoid tissue structure and development (Fig. 5e).

\section{EVL is highly expressed in lymphoid leukemia}

The genomic EVL/MIR342 locus was previously shown to be frequently methylated in colon cancer [38] and upregulated EVL expression was reported in breast cancer [39]. We then asked whether expression of EVL is associated with a specific subtype of leukemia. To address this, we reanalyzed the publicly available gene expression data set of 2096 leukemia samples of the MILE study (Microarray Innovations in LEukemia) from the ELN (European Leukemia Network). EVL was one of the top 100 differentially expressed genes between ALL and AML samples. Strikingly, we detected the highest expression of EVL in all subtypes of lymphoid leukemia, especially in T-ALL compared to healthy $\mathrm{BM}$ or myeloid malignancies (Fig. 6a). Within an independent data set of 120 CLL patients [40], EVL expression is associated with mutated IGHV ( $P=0.037$; Fig. 6 b). To get further insights into miR-342 expression in myeloid leukemia, we analyzed a small RNA sequencing data set of AML patients, grouped based on the mutational status of the AML driver gene FLT3, in comparison to healthy controls $(n=17)$ [41]. We observed increased miR-342 expression in AML samples, especially in patients without a FLT3 mutation, compared to healthy BM-derived mononuclear cells that did not reach statistical significance $(P=0.06$; Fig. $6 \mathrm{c})$. Analyzing the TCGA-LAML cohort of about 188 cases revealed that higher expression of mir-342 is associated with the morphological (FAB-) subtype M0 $(P=0.04)$ and the adverse risk category (according to ELN; $P=0.005$; Table S8). Molecularly, this analysis indicates that high mir-342 expression correlates with mutations in RUNX1 $(P=$ $0.009)$, and TP53 $(P=0.0006)$. In contrast, low mir-342 expression is significantly associated with mutated NPMI $(P<0.0001)$. These data suggest that the EVL/MIR342 locus may play a role in leukemia, however its potential prognostic impact needs further investigation.

\section{Discussion}

We here describe a genomic locus shared by a proteincoding gene and a miRNA that was identified by $\gamma \mathrm{RV}$ tags in human repopulating long-term HSCs of ten clinical gene therapy patients. Our data demonstrate that the proteincoding gene $E v l$ and its intronic miR-342 counterbalance each other in hematopoietic lineage differentiation in vitro, thereby maintaining a balanced generation of hematopoietic blood cells. Clustered retroviral therapeutic vector integrations into this genomic locus detected in the peripheral blood of patients several years after HSC gene therapy strongly hint towards expression and relevance of these genes in hematopoietic stem and progenitor cells. Previous studies focused either on EVL or miR-342 without considering their co-expression within the same locus.

On the molecular level, EVL was shown to play a pivotal role in actin cytoskeleton remodeling [26, 42] and homologous recombination. EVL is a member of the Ena/VASP family [43] and all three proteins are mainly involved in axon guidance, cell adhesion, and migration due to their actin-binding potential [44-47]. Homozygous Evl knockout mice are viable and fertile whereas the deletion of all three family members Ena/Vasp/Evl causes the development of brain defects and abnormal leukocyte migration [48]. Strongly in line with our results, showing the highest expression of EVL in B- and T-cell lineages, Lanier et al. detected a high expression of EVL in spleens and thymi of mice [44]. Moreover, we were able to show that the OE of EVL leads to the deregulation of pathways involved in Bcell and T-cell development and drives lymphopoiesis in vitro and in vivo. In contrast, miR-342 OE increases myeloid colony formation with a higher frequency of myelopoietic progenitors in vivo. In accordance with our data, recent studies demonstrated that miR-342 is a direct target of the transcription factor PU.1 leading to an accelerated all-trans retinoic acid-induced myeloid differentiation of APL blasts [49] and that miR-342 is expressed in human and murine macrophages playing an important role in proapoptotic signaling after IL-4 stimulation [50].

In contrast to the results of our study, it was suggested that miR-342 exerts a synergistic function with EVL [16] as it is co-expressed with its host gene [16, 51, 52]. Our subsequent investigation of promoter domains, active enhancer regions and transcription factor binding sites together with gene expression in hematopoietic cells revealed that miR-342 is indeed co-regulated with its host gene. This result is reinforced by the identification of miR342 as a downstream target of Notch1 [53] suppressed via Hes5 binding sites within the $E v l$ promoter [28]. We then tested the hypothesis whether increasing amounts of miR342 influence the cellular localization of EVL, however, we have no indication for that (data not shown), which argues for an alteration of differentiation regulating expression programs. Global gene expression profiling of $\mathrm{EVL}^{+}$or miR-342 ${ }^{+}$LSK cells strongly supports their molecular influence on lymphoid or myeloid lineage choice, respectively. We showed that simultaneous OE of both genes has a neutralizing effect on the hematopoietic lineage output. EVL abolished the myeloid-biased differentiation upon miR-342 expression, whereas the induction of miR-342 in EVL expressing LSK cells reduced lymphoid colony formation substantially. We demonstrate that the interaction of 
a.

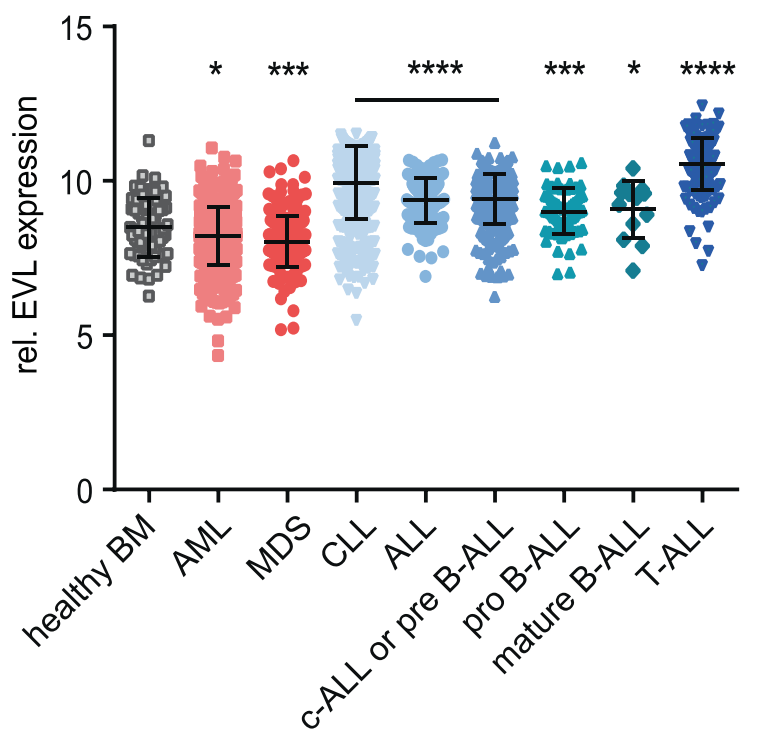

C.

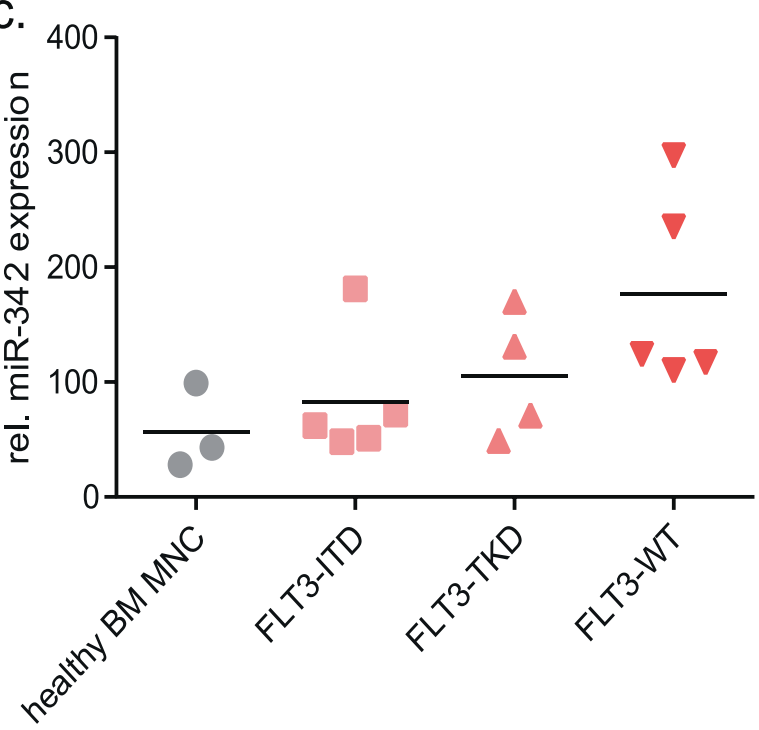

Fig. 6 EVL is significantly overexpressed in lymphoid leukemia compared to healthy bone marrow and myeloid malignancies. a Expression of EVL in 2096 patient samples with hematopoietic malignancies compared to healthy bone marrow (BM). AML acute myeloid leukemia, MDS myelodysplastic syndrome, CLL chronic lymphoid leukemia, ALL acute lymphoid leukemia. b Association of the expression level of EVL with immunoglobulin heavy chain gene

the host gene and its intronic miRNA is exerted by miR-342 mediated suppression of genes important in lymphopoiesis. In the same line, EVL driven miR-342 target genes may act as individual sponges, thereby suppressing the role of miR342 in myelopoiesis, which is supported by a reduced myeloid colony output of miR- $342^{+}$LSK cells compared to mock transduced cells in presence of a specific sponge vector (data not shown). This clearly establishes the antagonistic role of miR-342 towards EVL driven lineage determination in hematopoiesis. Interestingly, Gao et al. b.

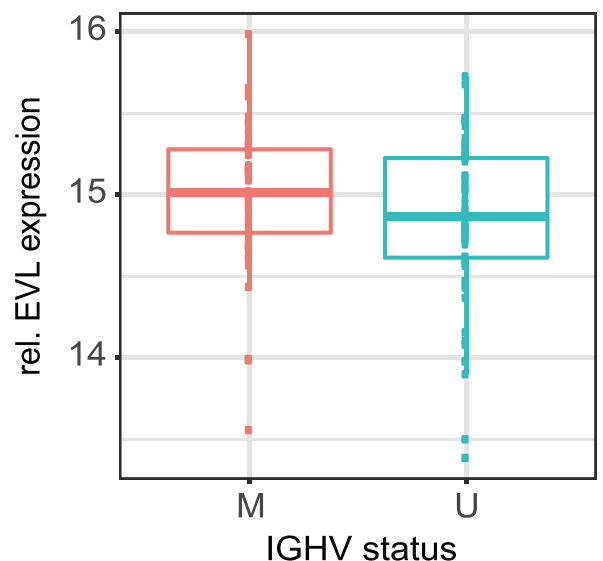

(IGHV) mutation status in 120 CLL samples. M mutated, U unmutated. c Relative miR-342-3p expression identified by small RNA-seq in the indicated AML samples with normal karyotype compared to healthy bone marrow-derived mononuclear cells (BM MNC). FLT3 FMS-related tyrosine kinase 3, ITD internal tandem duplications, TKD tyrosine kinase domain. $* P<0.05 ; * * P<0.01 ; * * * P<0.001$.

[28] showed a modulatory role of miR-342 also on neural lineage differentiation in the brain. MiR-342 OE in neural stem cells promoted the generation of intermediate neural progenitors and inhibited the differentiation of astrocytes. Phylogenomic analyses describe the importance of actin-associated processes for phagocytosis and that genes involved in actin filament formation, actin binding or nucleation are highly conserved throughout eukaryotes [54]. Therefore, it is tempting to speculate that conserved gene loci involved in actin regulation like the Evl/Mir342 locus 
have contributed to the development of innate and adaptive immune cells and are still relevant for their balanced generation.

We identified Tcf12 (Transcription Factor 12), a basic helix-loop-helix transcription factor, as one miR-342targeted mRNA expressed in LSK cells. Its function is important for developmental processes like T-lymphopoiesis and neurogenesis $[3,55]$. The disruption of the Tcf12 locus in hESCs followed by mesodermal and hematopoietic differentiation in vitro clearly demonstrated the failure to generate committed T-cell precursors without an impact on myeloid differentiation [56]. This failure in early T-cell development could be fully restored by ectopic expression of HEBcan, the canonical protein transcribed from the $T c f 12$ locus [56]. Remarkably, the deletion of HEB in mice led to a profound reduction of committed B-cells in vivo as well as in vitro due to a developmental defect within CLP [57], which is in line with the reduced B-cell colony formation upon OE of miR-342, further supporting that Tcf12 is a direct target of miR-342. Interestingly, TCF12 is also expressed in lymphoid malignancies [58], suggesting a role in B- and T-cell-derived leukemia. In addition, we identified Mitf and Irf8 as further miR-342 targets in hematopoietic cells. Importantly, Irf8 is significantly upregulated upon Evl OE in LSK cells, leading to the activation of downstream target molecules. In line with our data showing that miR342 , which targets Irf8, results in increased myeloid colony formation in vitro and in vivo, $\operatorname{Irf} 8$ knockout mice exhibit expansion of granulocytes and macrophages [59], and Scheller et al. showed an increased sensitivity of $I R F 8^{-/-}$ myeloid progenitors to G-CSF and GM-CSF leading to myeloid expansion [60]. Our results strongly suggest that IRF8 plays a major role in balanced lineage output driven by Evl/Mir342 via the IRF8/PU.1 axis. IRF8 has been shown to negatively regulate PU.1 [61], thereby controlling transcriptional programs for lymphoid or myeloid lineage specification by suppressing the other branch in return.

In summary, our data show that two hematopoietic differentiation programs are driven by one common and evolutionary highly conserved genetic locus. The interaction between EVL and miR-342 is important for hematopoietic differentiation by maintaining a balanced lineage output under steady-state conditions. This provides the blood system with a posttranscriptional mechanism to stabilize and quickly re-adjust lineage determination. Future studies are needed to show whether this is a common principle and whether additional genomic loci shared by host genes and intronic miRNAs have similar effects on balancing lineage determination also in other tissues and organs.

Acknowledgements The authors would like to thank Luigi Naldini (San Raffaele Telethon Institute for Gene Therapy, Milan, Italy) for providing lentiviral packaging plasmids and the transfer vector pCCL.
SIN.cPPT.PGK.IRES.eGFP.wPRE, Dieter Edbauer (German Center for Neurodegenerative Diseases, Munich, Germany) for providing the lentiviral transfer vector p.FCW, Béla Zimmer for cloning the miR342 expression vector, Julien Bethune for his advice regarding the AGO2 immunoprecipitation as well as Nina Hofmann and Sylvia Martin for their assistance in animal experiments or cell culture. We thank the microarray unit of the DKFZ Genomics and Proteomics Core Facility for providing the Illumina whole-genome expression Beadchips and related services. This work was done in Heidelberg (Germany) and was supported by the Deutsche Forschungsgemeinschaft (SFB873), by NCT3.0_2015.4 TransOnco and NCT3.0_2015.54 DysregPT. TJLL was supported by a scholarship of the Heinrich F.C. Behr Stiftung. PW, ESPE, and KL were supported by a scholarship of the Helmholtz International Graduate School for Cancer Research.

Author contributions Conceptualization: FH and HG; Methodology: FH, TJLL, ESPE, and PW; Investigation: FH, TJLL, ESPE, PW, AAW, TK, KL, SH, OZ, and AP; Formal Analysis: AH-W and JL; WritingOriginal Draft: FH and TJLL; Writing-Review \& Editing: FH, TJLL, ESPE, PW, CRB, and HG; Funding acquisition: FH, CRB, HG, and CvK; Resources: CvK, TZ, CK, and MS; Supervision: FH and HG.

Funding Open Access funding enabled and organized by Projekt DEAL.

\section{Compliance with ethical standards}

Conflict of interest CvK and MS are co-founders of GeneWerk GmbH.

Publisher's note Springer Nature remains neutral with regard to jurisdictional claims in published maps and institutional affiliations.

Open Access This article is licensed under a Creative Commons Attribution 4.0 International License, which permits use, sharing, adaptation, distribution and reproduction in any medium or format, as long as you give appropriate credit to the original author(s) and the source, provide a link to the Creative Commons license, and indicate if changes were made. The images or other third party material in this article are included in the article's Creative Commons license, unless indicated otherwise in a credit line to the material. If material is not included in the article's Creative Commons license and your intended use is not permitted by statutory regulation or exceeds the permitted use, you will need to obtain permission directly from the copyright holder. To view a copy of this license, visit http://creativecommons. org/licenses/by/4.0/.

\section{References}

1. Nerlov C, Graf T. PU.1 induces myeloid lineage commitment in multipotent hematopoietic progenitors. Genes Dev. 1998;12:2403-12.

2. Rieger MA, Hoppe PS, Smejkal BM, Eitelhuber AC, Schroeder T. Hematopoietic cytokines can instruct lineage choice. Science 2009;325:217-8.

3. Barndt R, Dai MF, Zhuang Y. A novel role for HEB downstream or parallel to the pre-TCR signaling pathway during alpha beta thymopoiesis. J Immunol. 1999;163:3331-43.

4. Shenoy A, Blelloch RH. Regulation of microRNA function in somatic stem cell proliferation and differentiation. Nat Rev Mol Cell Biol. 2014;15:565-76.

5. Bartel DP. MicroRNAs: genomics, biogenesis, mechanism, and function. Cell. 2004;116:281-97. 
6. Muljo SA, Ansel KM, Kanellopoulou C, Livingston DM, Rao A, Rajewsky K. Aberrant $\mathrm{T}$ cell differentiation in the absence of Dicer. J Exp Med. 2005;202:261-9.

7. Koralov SB, Muljo SA, Galler GR, Krek A, Chakraborty T, Kanellopoulou C, et al. Dicer ablation affects antibody diversity and cell survival in the B lymphocyte lineage. Cell. 2008;132:860-74.

8. Alemdehy MF, van Boxtel NG, de Looper HW, van den Berge IJ, Sanders MA, Cupedo T, et al. Dicer1 deletion in myeloidcommitted progenitors causes neutrophil dysplasia and blocks macrophage/dendritic cell development in mice. Blood. 2012;119:4723-30.

9. Lu J, Guo S, Ebert BL, Zhang H, Peng X, Bosco J, et al. MicroRNA-mediated control of cell fate in megakaryocyteerythrocyte progenitors. Dev Cell. 2008;14:843-53.

10. Xiao C, Calado DP, Galler G, Thai TH, Patterson HC, Wang J, et al. MiR-150 controls B cell differentiation by targeting the transcription factor c-Myb. Cell. 2007;131:146-59.

11. Guo S, Lu J, Schlanger R, Zhang H, Wang JY, Fox MC, et al. MicroRNA miR-125a controls hematopoietic stem cell number. Proc Natl Acad Sci USA. 2010;107:14229-34.

12. Haetscher N, Feuermann Y, Wingert S, Rehage M, Thalheimer FB, Weiser C, et al. STAT5-regulated microRNA-193b controls haematopoietic stem and progenitor cell expansion by modulating cytokine receptor signalling. Nat Commun. 2015;6:8928.

13. Mehta A, Zhao JL, Sinha N, Marinov GK, Mann M, Kowalczyk MS, et al. The microRNA-132 and microRNA-212 cluster regulates hematopoietic stem cell maintenance and survival with age by buffering FOXO3 expression. Immunity. 2015;42: 1021-32.

14. Lechman ER, Gentner B, van Galen P, Giustacchini A, Saini M, Boccalatte FE, et al. Attenuation of miR-126 activity expands HSC in vivo without exhaustion. Cell Stem Cell. 2012;11: 799-811.

15. Baskerville S, Bartel DP. Microarray profiling of microRNAs reveals frequent coexpression with neighboring miRNAs and host genes. RNA. 2005;11:241-7.

16. Lutter D, Marr C, Krumsiek J, Lang EW, Theis FJ. Intronic microRNAs support their host genes by mediating synergistic and antagonistic regulatory effects. BMC Genom. 2010;11:224.

17. Najafi-Shoushtari SH, Kristo F, Li Y, Shioda T, Cohen DE, Gerszten RE, et al. MicroRNA-33 and the SREBP host genes cooperate to control cholesterol homeostasis. Science. 2010;328:1566-9.

18. Rayner KJ, Suarez Y, Davalos A, Parathath S, Fitzgerald ML, Tamehiro N, et al. MiR-33 contributes to the regulation of cholesterol homeostasis. Science. 2010;328:1570-3.

19. Barik S. An intronic microRNA silences genes that are functionally antagonistic to its host gene. Nucleic Acids Res. 2008;36:5232-41.

20. Dill H, Linder B, Fehr A, Fischer U. Intronic miR-26b controls neuronal differentiation by repressing its host transcript, ctdsp2. Genes Dev. 2012;26:25-30.

21. Han J, Denli AM, Gage FH. The enemy within: intronic miR-26b represses its host gene, ctdsp2, to regulate neurogenesis. Genes Dev. 2012;26:6-10.

22. Braun CJ, Boztug K, Paruzynski A, Witzel M, Schwarzer A, Rothe M, et al. Gene therapy for Wiskott-Aldrich syndromelong-term efficacy and genotoxicity. Sci Transl Med. 2014;6:227ra33.

23. Deichmann A, Hacein-Bey-Abina S, Schmidt M, Garrigue A, Brugman $\mathrm{MH}, \mathrm{Hu} \mathrm{J}$, et al. Vector integration is nonrandom and clustered and influences the fate of lymphopoiesis in SCID-X1 gene therapy. J Clin Investig. 2007;117:2225-32.

24. Cattoglio C, Pellin D, Rizzi E, Maruggi G, Corti G, Miselli F, et al. High-definition mapping of retroviral integration sites identifies active regulatory elements in human multipotent hematopoietic progenitors. Blood. 2010;116:5507-17.
25. Wunsche P, Eckert ESP, Holland-Letz T, Paruzynski A, HotzWagenblatt A, Fronza R, et al. Mapping active gene-regulatory regions in human repopulating long-term HSCs. Cell Stem Cell. 2018;23:132-46.e9

26. Bournier O, Kroviarski Y, Rotter B, Nicolas G, Lecomte MC, Dhermy D. Spectrin interacts with EVL (Enabled/vasodilator-stimulated phosphoprotein-like protein), a protein involved in actin polymerization. Biol Cell. 2006;98:279-93.

27. Yu-Kemp HC, Kemp JP, Jr., Brieher WM. CRMP-1 enhances EVL-mediated actin elongation to build lamellipodia and the actin cortex. J Cell Biol. 2017;216:2463-79.

28. Gao F, Zhang YF, Zhang ZP, Fu LA, Cao XL, Zhang YZ, et al. miR342-5p regulates neural stem cell proliferation and differentiation downstream to notch signaling in mice. Stem Cell Rep. 2017;8:1032-45.

29. Herbst F, Ball CR, Tuorto F, Nowrouzi A, Wang W, Zavidij O, et al. Extensive methylation of promoter sequences silences lentiviral transgene expression during stem cell differentiation in vivo. Mol Ther. 2012;20:1014-21.

30. Castro F. High-throughput SNP-based authentication of human cell lines. Int J Cancer. 2013;132:308-314. https://doi.org/10. 1002/ijc. 27675.

31. Puck JM, Candotti F. Lessons from the Wiskott-Aldrich syndrome. N. Engl J Med. 2006;355:1759-61.

32. Thrasher AJ. WASp in immune-system organization and function. Nat Rev Immunol. 2002;2:635-46.

33. De Rijck J, de Kogel C, Demeulemeester J, Vets S, El Ashkar S, Malani N, et al. The BET family of proteins targets moloney murine leukemia virus integration near transcription start sites. Cell Rep. 2013;5:886-94.

34. LaFave MC, Varshney GK, Gildea DE, Wolfsberg TG, Baxevanis $\mathrm{AD}$, Burgess SM. MLV integration site selection is driven by strong enhancers and active promoters. Nucleic Acids Res. 2014;42:4257-69.

35. Cabezas-Wallscheid N, Klimmeck D, Hansson J, Lipka DB, Reyes A, Wang Q, et al. Identification of regulatory networks in HSCs and their immediate progeny via integrated proteome, transcriptome, and DNA methylome analysis. Cell Stem Cell. 2014;15:507-22.

36. Fournier M, Lebert-Ghali CE, Krosl G, Bijl JJ. HOXA4 induces expansion of hematopoietic stem cells in vitro and confers enhancement of pro-B-cells in vivo. Stem Cells Dev. 2012;21:133-42.

37. Alharbi RA, Pettengell R, Pandha HS, Morgan R. The role of HOX genes in normal hematopoiesis and acute leukemia. Leukemia. 2013;27:1000-8.

38. Grady WM, Parkin RK, Mitchell PS, Lee JH, Kim YH, Tsuchiya $\mathrm{KD}$, et al. Epigenetic silencing of the intronic microRNA hsamiR-342 and its host gene EVL in colorectal cancer. Oncogene. 2008;27:3880-8.

39. Hu LD, Zou HF, Zhan SX, Cao KM. EVL (Ena/VASP-like) expression is up-regulated in human breast cancer and its relative expression level is correlated with clinical stages. Oncol Rep. 2008;19:1015-20.

40. Dietrich S, Oles M, Lu J, Sellner L, Anders S, Velten B, et al. Drug-perturbation-based stratification of blood cancer. J Clin Investig. 2018;128:427-45.

41. Krakowsky RHE, Wurm AA, Gerloff D, Katzerke C, BrauerHartmann D, Hartmann JU, et al. miR-451a abrogates treatment resistance in FLT3-ITD-positive acute myeloid leukemia. Blood. Cancer J. 2018;8:36.

42. Lambrechts A, Kwiatkowski AV, Lanier LM, Bear JE, Vandekerckhove J, Ampe C, et al. cAMP-dependent protein kinase phosphorylation of EVL, a Mena/VASP relative, regulates its interaction with actin and $\mathrm{SH} 3$ domains. J Biol Chem. 2000;275:36143-51. 
43. Gertler FB, Niebuhr K, Reinhard M, Wehland J, Soriano P. Mena, a relative of VASP and Drosophila Enabled, is implicated in the control of microfilament dynamics. Cell. 1996;87:227-39.

44. Lanier LM, Gates MA, Witke W, Menzies AS, Wehman AM, Macklis JD, et al. Mena is required for neurulation and commissure formation. Neuron. 1999;22:313-25.

45. Reinhard M, Halbrugge M, Scheer U, Wiegand C, Jockusch BM, Walter U. The $46 / 50 \mathrm{kDa}$ phosphoprotein VASP purified from human platelets is a novel protein associated with actin filaments and focal contacts. EMBO J. 1992;11:2063-70.

46. Rottner K, Behrendt B, Small JV, Wehland J. VASP dynamics during lamellipodia protrusion. Nat Cell Biol. 1999;1:321-2.

47. Breitsprecher D, Kiesewetter AK, Linkner J, Vinzenz M, Stradal TE, Small JV, et al. Molecular mechanism of Ena/VASP-mediated actin-filament elongation. EMBO J. 2011;30:456-67.

48. Kwiatkowski AV, Rubinson DA, Dent EW, Edward van Veen J, Leslie JD, Zhang J, et al. Ena/VASP Is Required for neuritogenesis in the developing cortex. Neuron. 2007;56:441-55.

49. De Marchis ML, Ballarino M, Salvatori B, Puzzolo MC, Bozzoni I, Fatica A. A new molecular network comprising PU.1, interferon regulatory factor proteins and miR-342 stimulates ATRAmediated granulocytic differentiation of acute promyelocytic leukemia cells. Leukemia. 2009;23:856-62.

50. Czimmerer Z, Varga T, Kiss M, Vazquez CO, Doan-Xuan QM, Ruckerl D, et al. The IL-4/STAT6 signaling axis establishes a conserved microRNA signature in human and mouse macrophages regulating cell survival via miR-342-3p. Genome Med. 2016;8:63.

51. Ronchetti D, Lionetti M, Mosca L, Agnelli L, Andronache A, Fabris S, et al. An integrative genomic approach reveals coordinated expression of intronic miR-335, miR-342, and miR-561 with deregulated host genes in multiple myeloma. BMC Med Genom. 2008;1:37.

52. Marsico A, Huska MR, Lasserre J, Hu H, Vucicevic D, Musahl A, et al. PROmiRNA: a new miRNA promoter recognition method uncovers the complex regulation of intronic miRNAs. Genome Biol. 2013;14:R84.
53. Yan XC, Cao J, Liang L, Wang L, Gao F, Yang ZY, et al. miR$342-5 p$ is a notch downstream molecule and regulates multiple angiogenic pathways including notch, vascular endothelial growth factor and transforming growth factor beta signaling. J Am Heart Assoc. 2016;5:R84.

54. Yutin N, Wolf MY, Wolf YI, Koonin EV. The origins of phagocytosis and eukaryogenesis. Biol Direct. 2009;4:9.

55. Ravanpay AC, Olson JM. E protein dosage influences brain development more than family member identity. J Neurosci Res. 2008;86:1472-81.

56. Li Y, Brauer PM, Singh J, Xhiku S, Yoganathan K, ZunigaPflucker JC, et al. Targeted disruption of TCF12 reveals HEB as essential in human mesodermal specification and hematopoiesis. Stem Cell Rep. 2017;9:779-95.

57. Welinder E, Mansson R, Mercer EM, Bryder D, Sigvardsson M, Murre C. The transcription factors E2A and HEB act in concert to induce the expression of FOXO1 in the common lymphoid progenitor. Proc Natl Acad Sci USA. 2011;108:17402-7.

58. Haferlach T, Kohlmann A, Wieczorek L, Basso G, Kronnie GT, Bene MC, et al. Clinical utility of microarray-based gene expression profiling in the diagnosis and subclassification of leukemia: report from the International Microarray Innovations in Leukemia Study Group. J Clin Oncol. 2010;28: 2529-37.

59. Giese NA, Gabriele L, Doherty TM, Klinman DM, TadesseHeath L, Contursi C, et al. Interferon (IFN) consensus sequencebinding protein, a transcription factor of the IFN regulatory factor family, regulates immune responses in vivo through control of interleukin 12 expression. J Exp Med. 1997;186: 1535-46.

60. Scheller M, Foerster J, Heyworth CM, Waring JF, Lohler J, Gilmore GL, et al. Altered development and cytokine responses of myeloid progenitors in the absence of transcription factor, interferon consensus sequence binding protein. Blood. 1999;94:3764-71.

61. Wang H, Lee CH, Qi C, Tailor P, Feng J, Abbasi S, et al. IRF8 regulates B-cell lineage specification, commitment, and differentiation. Blood. 2008;112:4028-38.

\section{Affiliations}

\section{Friederike Herbst $\mathbb{1}^{1}$ - Tonio J. L. Lang ${ }^{1,2}$ - Elias S. P. Eckert ${ }^{1,3}$ - Peer Wünsche ${ }^{1,3}$ - Alexander A. Wurm ${ }^{4,5,6}$. Tim Kindinger ${ }^{1} \cdot$ Karin Laaber $\mathbb{D}^{1,3} \cdot$ Shayda Hemmati ${ }^{1,3} \cdot$ Agnes Hotz-Wagenblatt $\mathbb{D}^{7} \cdot$ Oksana Zavidij ${ }^{1}$. Anna Paruzynski ${ }^{8}$. Junyan $\mathrm{Lu}^{9} \cdot$ Christof von Kalle $\mathrm{e}^{10,11,12} \cdot$ Thorsten Zenz $\mathbb{D}^{13} \cdot$ Christoph Klein $\mathbb{1}^{14}$. Manfred Schmidt ${ }^{10,11} \cdot$ Claudia R. Ball $^{5,6,15} \cdot$ Hanno Glimm ${ }^{1,5,6,15}$}

1 Translational Functional Cancer Genomics, National Center for Tumor Diseases (NCT) and German Cancer Research Center (DKFZ), 69120 Heidelberg, Germany

2 Charité - Universitätsmedizin Berlin, corporate member of Freie Universität Berlin and Humboldt-Universität zu Berlin, Department of Hematology, Oncology and Tumorimmunology, Augustenburger Platz 1, 13353 Berlin, Germany

3 Faculty of Biosciences, University Heidelberg, 69120 Heidelberg, Germany

4 Mildred Scheel Early Career Center, National Center for Tumor Diseases Dresden (NCT/UCC), Medical Faculty and University Hospital Carl Gustav Carus, Technische Universität Dresden, 01307 Dresden, Germany
5 Department of Translational Medical Oncology, National Center for Tumor Diseases (NCT) Dresden and German Cancer Research Center (DKFZ), 01307 Dresden, Germany

6 Center for Personalized Oncology, National Center for Tumor Diseases (NCT) Dresden and University Hospital Carl Gustav Carus Dresden at TU Dresden, 01307 Dresden, Germany

7 Omics IT and Data Management Core Facility, German Cancer Research Center (DKFZ), 69120 Heidelberg, Germany

8 BioNTech Manufacturing GmbH, 55131 Mainz, Germany

9 European Molecular Biology Laboratory (EMBL), Genome Biology Unit, 69117 Heidelberg, Germany

10 Department of Translational Oncology, National Center for Tumor Diseases (NCT) and German Cancer Research Center (DKFZ), 69120 Heidelberg, Germany 
11 GeneWerk GmbH, 69120 Heidelberg, Germany

12 German Cancer Consortium (DKTK), 69120

Heidelberg, Germany

13 Department of Medical Oncology and Haematology, University Hospital Zurich \& University of Zurich, 8091 Zurich, Switzerland
14 Department of Pediatrics, Dr. von Hauner Children's Hospital, University Hospital, LMU Munich, 80337 Munich, Germany

15 German Cancer Consortium (DKTK), 01307 Dresden, Germany 